\title{
Neurological complications of COVID-19: a preliminary review
}

\author{
A. Pryce-Roberts ${ }^{1} \cdot$ M. Talaei ${ }^{1} \cdot$ N. P. Robertson ${ }^{1}$
}

Published online: 3 June 2020

(c) The Author(s) 2020

\section{Introduction}

Coronavirus disease 19 (COVID-19) is a highly transmissible viral infection caused by severe acute respiratory syndrome coronavirus 2 (SARS-CoV-2), which emerged in Wuhan, China and has since spread rapidly around the world. Coronaviruses are so named for their characteristic crown-like appearance and are widespread in nature infecting several different species causing mainly respiratory and enteric pathology [1].

SARS-CoV-2 is the seventh coronavirus known to infect humans; whereas four are endemic (HKU1, NL63, OCw43 and 229E) and cause a considerable share of mild upper and lower respiratory tract infections, SARS-CoV, MERSCoV and SARS-CoV-2 can cause severe disease [2]. The spectrum of pathology in COVID-19 is wide and ever increasing with a burgeoning number of case reports and case series published on a daily basis that provide some preliminary insights to the disease and its complications. However, it is certain that larger case series and population-based studies will follow, as is the usual pattern with novel diseases. In this month's journal club, and in a break from the normal format, we have attempted to provide an early snapshot of the current literature describing neurological manifestations of this novel disease in a very fast moving subject area, but in the certain knowledge that this will be superseded in due course by more comprehensive studies.

N. P. Robertson

robertsonnp@cardiff.ac.uk

1 Division of Psychological Medicine and Clinical Neuroscience, Department of Neurology, Cardiff University, University Hospital of Wales, Heath Park, Cardiff CF14 4XN, UK

\section{Neurological manifestations of COVID-19}

As of the 1st May 2020, there have been 52 data papers comprising case reports or case series and 27 reviews including editorials/position papers. In January 2020, a retrospective, single-center study on 99 patients diagnosed with SARSCOV-2 pneumonia at a hospital in Wuhan identified confusion and headache in $9 \%$ and $8 \%$, respectively [3]. A recently published retrospective, observational study from Wuhan in 214 patients with RT-pPCR confirmed COVID-19 reported $36 \%$ had neurologic manifestations [4]; central nervous system (CNS) 25\%, peripheral nervous system (PNS) $9 \%$ and skeletal muscle injury $11 \%$. In patients with CNS manifestations, the most commonly reported symptoms were dizziness (17\%) and headache (13\%). In patients with PNS symptoms, the commonest were dysgeusia $6 \%$ and hyposmia $5 \%$. CNS symptoms were also more commonly identified in those with severe disease with associated thrombocytopenia, increased urea levels and lymphopenia.

A study of patients admitted to intensive care units (ITU) has also emphasized the frequency of neurological features [5]. In an observational series of 58 of 64 consecutive patients admitted to hospital with acute respiratory distress syndrome (ARDS) secondary to COVID-19, 40 (69\%) displayed agitation and 39 (67\%) exhibited diffuse corticospinal tract signs with enhanced tendon reflexes, ankle clonus, and bilateral extensor plantar reflexes. Of the patients who had been discharged by the time of publication, 15/45 (33\%) were noted to have a dysexecutive syndrome consisting of inattention, disorientation, or poorly organized movements in response to command. However, in the seven patients who underwent CSF analysis, RT-PCR for SARS-CoV-2 was negative in all cases.

In addition to neurological symptoms accompanying the classical respiratory presentation of COVID-19, case reports and series have identified a wide range of acute clinical neurological syndromes which largely fall into three main categories: stroke, Guillain-Barré Syndrome (GBS) and variants and meningoencephalitis/encephalopathy/encephalitis. There are also a smaller numbers of studies reporting 
epilepsy $(n=3)$, acute disseminated encephalomyelitis (ADEM) and acute myelitis. In addition there are reports of psychiatric and neuropsychiatric presentations which are not reviewed in this article.

\section{Stroke}

Of 12 reports published up to the 1st May 2020, 5 were single case reports, 4 were case series (reporting between 2 and 6 cases), 2 were observational studies describing the incidence of CVA in patients positive for RT-PCR for SARS$\mathrm{CoV}-2$, whilst a further study is a meta-analysis of four studies which reported that patients with a history of stroke have an increased odds ratio of 2.5 for developing severe COVID19 [6]. The two observational studies describe case series of patients with COVID-19. In the first retrospective study of 221 patients, 11 (5\%) developed acute ischemic stroke, $1(0 \cdot 5 \%)$ cerebral venous sinus thrombosis (CVST), and 1 $(0 \cdot 5 \%)$ cerebral hemorrhage [7]. In the second prospective study of 288 patients, ischemic stroke was diagnosed in 9 (2.5\%) of included patients [8].

These 9 case reports and case series together detail a total of 21 patients; [9-17] 16 males and 5 females. Age was precisely stated in 20 cases and was a mean of 59.8 years (male63.1, female-49.8), although may be artificially depressed by the case series of young strokes [12]. The majority (19) were ischemic strokes, although three underwent hemorrhagic transformation, whilst 2 were hemorrhagic at onset. Of the 19 ischemic cases, 8 had multiple infarcts. Thirteen patients had thrombus detectable on imaging; 6 within the middle cerebral, 2 posterior cerebral, three vertebral/basilar and 1 internal carotid. A further case had multiple sites of thrombus. Of the remainder, four were consistent with large-vessel occlusions but the presence of thrombus was not explicitly stated; the remaining two were lacunar infarcts. Several of the reports note other thrombotic events; four patients had pulmonary emboli (three bilateral), one patient presented with myocardial infarction and ischemic limbs, while ischemic limbs and renal infarction were also noted in other cases. Of the 19 patients with ischemic stroke, four patients died, six were still supported on an intensive care unit (ICU), one remained on a stroke unit, two were at a stroke rehabilitation facility and five made a good recovery. Outcome in one case was not stated.

Also of interest, significantly elevated D-Dimer levels $(\geq 1000 \mu \mathrm{g} / \mathrm{L}$ ) were reported in 14 cases, of which nine had anticardiolipin antibodies and lupus anticoagulant tests with variable results. In a series of six patients from the UK, one patient had medium titre for IgM anticardiolipin antibodies and lupus anticoagulant was positive in five of six cases [10]. However in a series of three patients from Beijing, all patients were positive for anticardiolipin $\operatorname{IgA}$ antibodies as well as anti- $\beta 2$-glycoprotein I IgA and $\operatorname{IgG}$ antibodies but lupus anticoagulant was not detected in any patients [13].

\section{Guillain-Barré Syndrome and variants}

Of 8 reports published by 1 st of May 2020, 6 were single case reports [18-23] and 2 were case series $(n=2-5)$ $[24,25]$ describing a total of 13 para-COVID-19 or post COVID-19 GBS (10 males, 3 females, mean age 58.9; male 55.8 , female 69.7). Eleven were positive for SARS-COV-2 RT-qPCR from oropharyngeal swabs. Two were RT-qPCR negative; one presented 24 days after COVID-19 respiratory symptoms but with typical CT chest abnormalities [19] and one where the GBS presentation preceded respiratory symptoms by 8 days [23].

Eleven cases presented with progressive a flexic flaccid tetraparesis of whom five developed bilateral facial weakness and seven required intubation and ventilation. Two cases presented with a Miller Fisher variant [25]. Neurological symptoms developed between 3 and 28 days after emergence of respiratory symptoms at a median interval of 11 days. Twelve of the 13 cases were treated with IVIG. At 4 weeks, two patients required ongoing mechanical ventilation in ITU, three were undergoing rehabilitation, four had been discharged and one had died. Outcomes for the remaining three patients are unknown.

Lumbar puncture was performed on 11 patients from day 1 to 5 of neurological symptoms; white cells were typically normal or mildly raised $(1-9 / \mu \mathrm{L}$ in 5 cases), protein was within normal range in 3 cases and $>45 \mathrm{mg} / \mathrm{dL}$ in the remaining 8 cases (range $48-193 \mathrm{mg} / \mathrm{dL}$; average $104.6 \mathrm{mg} /$ dL). Eight had CSF tested for SARS-COV2 PCR all of which were negative. Ten had neurophysiological examinations between day 3 and 12 of neurological symptoms; 6 showed changes consistent with demyelination and 4 with axonal variant GBS. Anti-ganglioside antibodies results were reported in only four; three were negative whilst a patient with Miller Fisher presentation had positive GD1b antibody but negative GQ1b [25].

\section{Encephalitis, meningoencephalitis and encephalopathy}

Of the 8 reports published up to May 1st 2020, 7 were single case reports [26-32] and one a case series $(n=2)$ [33] in the context of oropharyngeal swabs or CSF positive for SARS-Cov-2 using RT-qPCR. Of the 9 cases, 5 were females and 3 were males; one did not specify gender; average age 55.5; male 42.0, female 60.8. Clinical presentations were generally consistent with the fever and confusion commonly described. Meningism was noted in 
three, headache in four, seizures in three and three exhibited frontal release signs. A single case of acute necrotizing encephalopathy in a confirmed COVID-19 patient was also reported [28].

Imaging failed to demonstrate acute changes in the majority. However, in one case hyperintense signal in the right mesial temporal lobe and hippocampus was noted [30] whilst hemorrhagic rim enhancing lesions within the bilateral thalami, medial temporal lobes and subinsular regions was reported in the case of acute necrotizing encephalopathy [28]. EEG was performed in two of the cases presenting with seizures as well as one other with encephalopathy [29]; all showed generalized slowing.

Of the nine published cases, seven tested CSF for SARSCov-2; two noted that CSF testing was not available at their institution [28, 31]. Two cases had positive CSF PCR for SARS-Cov-2 [26, 30], five were negative [27, 29, 32, 33]. The majority of patients were treated with broad spectrum antibiotics and antiviral medications. Two recovered spontaneously within 4 days [33] and one showed little response to antivirals and hydroxychloroquine but appeared to make a dramatic recovery on commencement of high dose steroids and was discharged within 2 weeks of presentation [29]. Two others who showed little response to initial treatment improved with a mannitol infusion [27] and hydroxychloroquine [31], respectively. Two patients remained in ITU [30, $32]$ and no clear outcome was documented in a further two cases $[26,28]$.

\section{Comment}

It is becoming apparent that neurological complications of COVID-19 are relatively common together with a suggestion that they are seen more frequently with increasing disease severity. In common with previous pandemics including Zika and SARS-CoV, stroke, GBS and meningoencephalitis are emerging as the commonest specific neurological complications but causality remains difficult to prove. Stroke in particular may have a plausible biological mechanism whilst GBS and meningoencephalitis have a compelling temporal relationship and are likely to represent parainfectious phenomenon. However, whether they are specific to COVID19 or whether they simply represent the consequence of a burden of infection remains unclear. In addition cognitive impairment is also emerging as a common sequelae in COVID-19 patients discharged from ITU and we can expect an increase in referrals to memory clinics in the short to medium term. Only longer term epidemiological studies will clarify some of these issues and will need to be supported by research on the neurotropic nature of the virus and biological mechanisms.
Open Access This article is licensed under a Creative Commons Attribution 4.0 International License, which permits use, sharing, adaptation, distribution and reproduction in any medium or format, as long as you give appropriate credit to the original author(s) and the source, provide a link to the Creative Commons licence, and indicate if changes were made. The images or other third party material in this article are included in the article's Creative Commons licence, unless indicated otherwise in a credit line to the material. If material is not included in the article's Creative Commons licence and your intended use is not permitted by statutory regulation or exceeds the permitted use, you will need to obtain permission directly from the copyright holder. To view a copy of this licence, visit http://creativecommons.org/licenses/by/4.0/.

\section{References}

1. Vabret A, Dina J, Brison E et al (2009) Coronavirus humains (HCoV). Pathol Biol 57:149-160

2. Corman VM, Muth D, Niemeyer D et al (2018) Hosts and sources of endemic human coronaviruses. Adv Virus Res 100:163-188

3. Chen N, Zhou M, Dong X et al (2020) Epidemiological and clinical characteristics of 99 cases of 2019 novel coronavirus pneumonia in Wuhan, China: a descriptive study. Lancet 395:507-513

4. Mao L et al (2020) Neurologic manifestations of hospitalized patients with coronavirus disease 2019 in Wuhan, China. JAMA Neurol. https://doi.org/10.1001/jamaneurol.2020.1127

5. Helms J, Kremer S, Merdji H et al (2020) Neurologic features in severe SARS-CoV-2 infection. N Engl J Med. https://doi. org/10.1056/NEJMc2008597

6. Aggarwal G, Lippi G, Michael Henry B (2020) Cerebrovascular disease is associated with an increased disease severity in patients with Coronavirus Disease 2019 (COVID-19): a pooled analysis of published literature. Int J Stroke 1747493020921664

7. Li Y et al (2020) Acute cerebrovascular disease following COVID-19: a single center, retrospective, observational study. SSRN Electron J. https://doi.org/10.2139/ssrn.3550025 (Published online April 10, 2020)

8. Lodigiani C, Iapichino G, Carenzo L et al (2020) Venous and arterial thromboembolic complications in COVID-19 patients admitted to an academic hospital in Milan, Italy. Thromb Res 191:9-14

9. González-Pinto T, Luna-Rodriguez A, Moreno-Estabanez A et al (2020) Emergency room neurology in times of COVID19: malignant ischemic stroke and SARS-COV2 infection. Eur J Neurol. https://doi.org/10.1111/ene.14286

10. Beyrouti R, Adams ME, Benjamin L et al (2020) Characteristics of ischaemic stroke associated with COVID-19. J Neurol Neurosurg Psychiatry. https://doi.org/10.1136/jnnp.2020.323586

11. Al Saiegh F, Ghosh R, Liebold A et al (2020) Status of SARSCoV-2 in cerebrospinal fluid of patients with COVID-19 and stroke. J Neurol Neurosurg Psychiatry. https://doi.org/10.1136/ jnnp-2020-323522

12. Oxley TJ, Mocco J, Majidi S et al (2020) Large-vessel stroke as a presenting feature of Covid-19 in the young. N Engl J Med. https://doi.org/10.1056/NEJMc2001272

13. Zhang Y, Xiao M, Zhang S et al (2020) Coagulopathy and antiphospholipid antibodies in patients with Covid-19. N Engl J Med 38:1-3

14. Lushina N, Kuo JS, Shaikh HA (2020) Pulmonary, cerebral, and renal thromboembolic disease associated with COVID19 Infection. Radiology. https://doi.org/10.1148/radiol.20202 01623 (published online ahead of print, 2020, Apr 23) 
15. Zhai P, Ding Y, Li Y (2020) The impact of COVID-19 on ischemic stroke: a case report. Res Sq. https://doi.org/10.21203 /RS.3.RS-20393/V1

16. Sharifi-Razavi A, Karimi N, Rouhani N (2020) COVID19 and intracerebral haemorrhage: causative or coincidental? New Microbes New Infect. https://doi.org/10.1016/j. nmni.2020.100669

17. Moshayedi P, Ryan TE, Mejia LLP et al (2020) Triage of acute ischemic stroke in confirmed COVID-19: large vessel occlusion associated with coronavirus infection. Front Neurol 11:353. https://doi.org/10.3389/fneur.2020.00353

18. Alberti P, Beretta S, Piatti M et al (2020) Guillain-Barré syndrome related to COVID-19 infection. Neurol Neuroimmunol Neuroinflammation 7:e741

19. Padroni M, Mastrangelo V, Asioli GM et al (2020) GuillainBarré syndrome following COVID-19: new infection, old complication? J Neurol. https://doi.org/10.1007/s00415-020-09849 $-6$

20. Virani A, Rabold E, Hanson T et al (2020) Guillain-Barré syndrome associated with SARS-CoV-2 infection. IDCases 20:e00771

21. Camdessanche JP, Morel J, Pozzetto B et al (2020) COVID-19 may induce Guillain-Barré syndrome. Rev Neurol (Paris). https ://doi.org/10.1016/j.neurol.2020.04.003

22. Sedaghat Z, Karimi N (2020) Guillain Barre syndrome associated with COVID-19 infection: a case report. J Clin Neurosci. https:// doi.org/10.1016/j.jocn.2020.04.062

23. Zhao H, Shen D, Zhou H et al (2020) Guillain-Barré syndrome associated with SARS-CoV-2 infection: causality or coincidence? Lancet Neurol 19:383-384

24. Toscano G, Palmerini F, Ravaglia S et al (2020) Guillain-Barré syndrome associated with SARS-CoV-2. N Engl J Med. https://
doi.org/10.1056/NEJMc2009191 (published online ahead of print, 2020 Apr 17)

25. Gutiérrez-Ortiz C, Mendez A, Rodrigo-Rey S et al (2020) Miller Fisher Syndrome and polyneuritis cranialis in COVID-19. Neurology. https://doi.org/10.1212/WNL.0000000000009619

26. Zhou L, Zhang M, Wang J et al (2020) Sars-Cov-2: underestimated damage to nervous system. Travel Med Infect Dis. https:// doi.org/10.1016/j.tmaid.2020.101642

27. Ye M, Ren Y, Lv T (2020) Encephalitis as a clinical manifestation of COVID-19. Brain Behav Immun. https://doi.org/10.1016/j. bbi.2020.04.017

28. Poyiadji N, Shahin G, Noujaim D et al (2019) COVID-19-associated acute hemorrhagic necrotizing encephalopathy: CT and MRI features. Imaging Radiol 6:5-10

29. Pilotto A, Odolini S, Stefano Masciocchi S et al (2020) Steroidresponsive severe encephalopathy in SARS-CoV-2 infection. medRxiv. https://doi.org/10.1101/2020.04.12.20062646

30. Moriguchi T, Harri N, Goto J et al (2020) A first Case of Meningitis/Encephalitis associated with SARS-Coronavirus-2. Int J Infect Dis 94:55-58

31. Duong L, Xu P, Liu A (2020) Meningoencephalitis without respiratory failure in a young female patient with COVID-19 infection in Downtown Los Angeles, early April 2020. Brain Behav Immun. https://doi.org/10.1016/j.bbi.2020.04.024

32. Filatov A, Sharma P, Hindi F, Espinosa PS (2020) Neurological complications of coronavirus disease (COVID-19): encephalopathy. Cureus 12(3):e7352. https://doi.org/10.7759/cureus.7352

33. Bernard-Valnet R, Pizzarotti B, Anichini A et al (2020) Two patients with acute meningo-encephalitis concomitant to SARS-CoV-2 infection. medRxiv. https://doi. org/10.1101/2020.04.17.20060251 